\title{
ANALYTICAL REPRESENTATION OF CHARGE-DENSITY PROFILE AT A JELLIUM SURFACE COVERED WITH THE ALKALI METAL SUBMONOLAYERS*
}

\author{
J.M. ROGOWSKA, K.F. WOJCIECHOWSKI \\ Institute of Experimental Physics, University of Wrocław \\ Cybulskiego 36, 50-205 Wrocław, Poland \\ AND H. BogdanóW \\ Institute of Informatics, University of Wroclaw \\ Przesmyckiego 20, 50-151 Wroclaw, Poland \\ (Received Augus( 2, 1990)
}

\begin{abstract}
A simple trial function for the electron density profiles at the surface of jellium covered by a submonolayer of alkali adatoms is proposed. The values of work function changes due to adsorption, computed by use of this function, are in good agreement with experimental data. It is shown also that the proposed earlier trial functions for the electron density profiles at the metal surface covered by metallic adlayers, give a wrong sequence of surface concentration of adatoms, at which the minimum of work function for different adsorbates occurs.
\end{abstract}

PACS numbers: 77.30.+y

\section{Introduction}

The surface electron density profile (SEDP) determined numerically by Lang and Kohn [1] for a clean metal surface, is often approximated by simple trial functions [2]. The same can be said of the SEDP of an adsorbate-metal systems. For such system analytical models were proposed by Yamauchi and Kawabe [3]

*This work was supported by the Polish Academy of Sciences, Problem MR I 9, and the Ministry of Sciences and Higher Education the Project CPBP 01.08.A2. 
and also by Bigun $[4,5]$. When we use, however, these models for the calculation of the work function (WF) changes $\Delta \varphi$ due to alkali atoms adsorption, we arrive at a contradiction with the experimental data. Namely, the experimentally observed sequence of the surface concentration $N_{m}^{-}$of adatoms at which the minimum $\Delta \varphi_{m}$ of WF occurs is reversed in relation to the calculated one (Fig. 2, Section 3). The calculated $\Delta \varphi_{m}$ values also decrease too rapidly with the thickness $d$ of the adsorbate layer [6] in comparison with that observed experimentally and that calculated by Lang [6] (Fig. 1, Section 3).

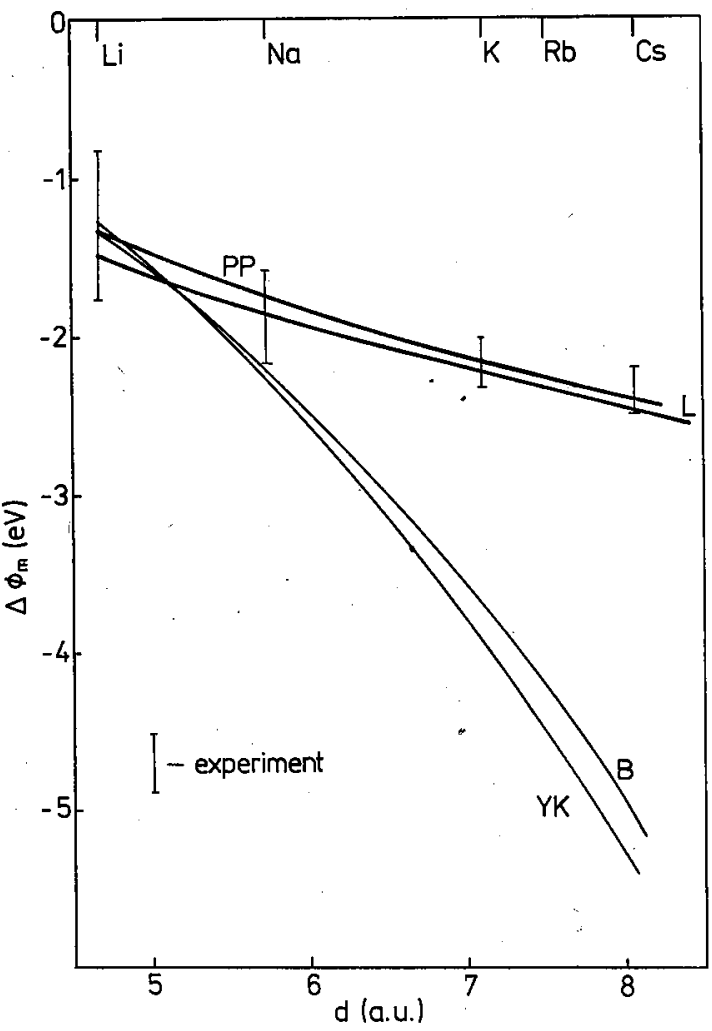

Fig. 1. The minimum of the WF change, $\Delta \varphi_{m}(N)$, for the metal $\left(r_{s}=2\right)$ - alkali metal adlayer system versus the thickness $d$ of the adlayer. The curve labelled PP is obtained from Eq. (18). Curves labelled: L, B and YK are obtained employing the SEDP described in $[6,5,3]$ respectively. The experimental points are taken from references cited in [6] for alkali metal adsorption on W(110).

To avoid the discrepancy between the results following from the models [3-5] and those from the experiment we propose another trial electron density profiles for the adsorbate-metal system, which will be considered in the framework of the jellium model. 
This paper is organized as follows: Section 2 gives the proposed model of SEDP, necessary assumptions and definitions. Section 3 comparises the findings of the proposed model with the experimental data and discusses the obtained results.

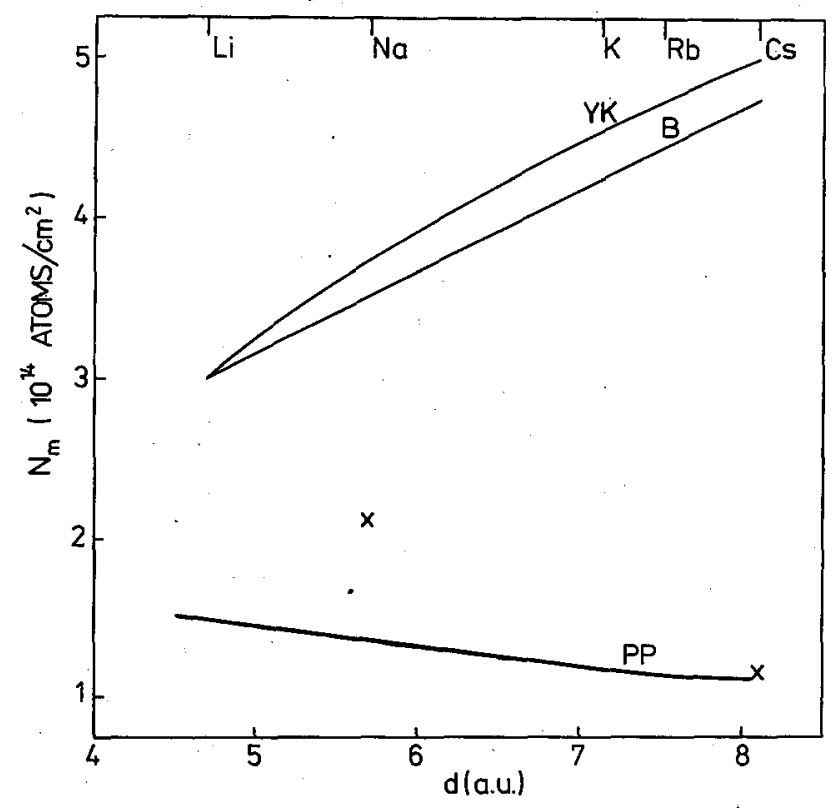

Fig. 2. The values of the surface concentration $N_{\mathrm{m}}$, at which the minimum of WF occurs, versus the thickness $d$ of the adsorbate layer on substrate with $r_{\mathrm{s}}=2$. The curve labelled PP is obtained from our model. Curves labelled $B$ and YK are obtained from SEDPs described in $[5,3]$, respectively. The crosses show Lang's results [6].

\section{Model of the trial SEDP}

For an adsorbate-metallic-substrate-system, we employ the core-charge density distribution $n_{+}(x)$ proposed by Lang [6]

$$
n_{+}(x)=\left\{\begin{array}{lll}
\bar{n} & \text { for } & x \leq 0 \\
n_{a} & \text { for } & 0<x \leq d, \\
0 & \text { for } & x>d .
\end{array}\right.
$$

The substrate, with the bulk electron density $\bar{n}=3\left(4 \pi r_{\mathrm{s}}^{3}\right)^{-1}$ occupies the semi-infinite half-space: $-\infty \leq x \leq 0$, where the $x$-axis is perpendicular to the metal surface, and the adsorbate layer of finite thickness $d$ is located on the surface of the substrate and has core-charge density equal to $n_{\mathrm{a}}(\theta)$, where $\theta$ is the degree of 
surface coverage (see App. I). In the present paper we shall use the values of $d$ assumed by Lang [6] which are independent of $\theta$.

We must point out that the above model has a physical meaning only if the coverage is large enough to start the metallization of submonolayers [7].

For the simulation of SEDP of the metal-adsorbate system, Yamauchi and Kawabe [3] and then also Bigun [4,5] employed Smoluchowski's [8] type of trial function, which for $x>0$ has a form: $B \exp (-\beta x)$, where $B$ and $\beta$ are positive constants. Such a function is not proper, however, to simulate the SEDP in the region of adsorbed slab. Namely, as it was shown by numerical calculation done by Lang [6], the SEDP of adsorbed slab is a convex function for $0 \leq x \leq d$. To regard this property of SEDP we propose the following trial function:

$$
\frac{n(x)}{\bar{n}}= \begin{cases}f_{1}(x)=F_{1}(x), & x \leq x_{0}, \\ f_{1}(x)+f_{2}(x)=F_{2}(x), & x>x_{0},\end{cases}
$$

where functions $f_{1}(x)$ and $f_{2}(x)$ describe the SEDP of metallic substrate and of adsorbed slab, respectively. The $x_{0}$ denotes the sticking point at which $F_{1}(x)=$ $F_{2}(x)$ and $\left.F_{1}^{\prime}(x)\right|_{x=x_{0}}=\left.F_{2}^{\prime}(x)\right|_{x=x_{0}}$.

For $f_{1}(x)$ we adopt the function proposed by Perdew [9]:

$$
f_{1}(x)= \begin{cases}1-b e^{c \alpha\left(x-x_{0}\right)} & \text { for } x \leq x_{0} \\ b_{1} e^{-c\left(x-x_{0}\right)} & \text { for } x>x_{0}\end{cases}
$$

where

$$
\begin{gathered}
b_{1}=1-b, \\
c=\gamma k_{\mathrm{s}}, \\
\alpha=b_{1} / b, \\
x_{0}=(2 b-1) / c b_{1} .
\end{gathered}
$$

For the function $f_{2}(x)$ we use the following expression:

$$
f_{2}(x)=p\left(x-x_{0}\right) \mathrm{e}^{-q\left(x-x_{0}\right)}, x \geq x_{0} .
$$

In Eq. (4b) $\gamma$ denotes [9] a variational parameter, and $k_{\mathrm{s}}^{2}=4 k_{\mathrm{F}} / \pi$, where $k_{\mathrm{s}}^{-1}$ is the Thomas-Fermi screening length, and $k_{\mathrm{F}}$ is the Fermi momentum $\left(\bar{n}=k_{\mathrm{F}}^{3} / 3 \pi^{2}\right)$. In Eq. (5) $p$ and $q$ denote the positive parameters. The way of their determination will be described later.

The trial function (2) with (3) and (5) has to satisfy the electric charge neutrality condition (ChNC) [10]:

$$
\int_{-\infty}^{+\infty} n_{\mathrm{t}}(x) \mathrm{d} x=0
$$

where

$$
n_{\mathrm{t}}(x)=n(x)-n_{+}(x)
$$


is the total charge density, connected with the electrostatic potential $\Phi(x)$ by the Poisson equation*

$$
\Phi(x)=\Phi(-\infty)+4 \pi \int_{-\infty}^{x} \mathrm{~d} x^{\prime}\left(x^{\prime}-x\right) n_{\mathrm{t}}\left(x^{\prime}\right)
$$

The ChNC applied to the function determined by Eqs. (3) and (5) gives the relation:

$$
p=s q^{2} d,
$$

where $s$ characterizes the surface concentration $N_{\mathrm{a}}$ of adatoms

and

$$
N_{\mathrm{a}}=N \times 10^{14} \mathrm{~cm}^{-2}=n_{\mathrm{a}} d
$$

$$
s=n_{\mathrm{a}} / \bar{n}=0.01172 N r_{\mathrm{s}}^{3} d^{-1} .
$$

In Eqs. (10-11) $N$ denotes the number of adatoms per unit area of the metal substrate (for other details see Appendix 1).

The parameters $\gamma$ and $q$ have been determined by the minimum of the surface energy $\sigma$ condition [10]:

$$
\frac{\partial \sigma\left|f_{1}(\gamma)\right|}{\partial \gamma}=0,\left.\quad \frac{\partial \sigma\left|f_{2}(q)\right|}{\partial q}\right|_{N=\text { const }}=0
$$

For the surface energy we use the sum of three components: $\sigma_{1}$ - the kinetic and the exchange-correlation energies of electron gas, where the correlation energy $\varepsilon_{c}$ is given by Wigner's expression (see Ref. 20 in [11]), $\sigma_{2}$ - the electrostatic energy of the system of charges, and $\sigma_{3}-$ the second order density gradient contributions to the surface kinetic energy [10].

After some algebra, one can obtain

$$
\frac{\sigma}{\bar{n}}=\frac{\sigma_{1}+\sigma_{2}+\sigma_{3}}{\bar{n}}=\Gamma\left(\gamma, r_{\mathrm{s}}\right)+Q\left(q, r_{\mathrm{s}}\right)
$$

where the functions $\Gamma$ and $Q$ are given in Appendix 2.

The values of the parameters $b\left(r_{\mathrm{s}}=2\right)$ and $\gamma\left(r_{\mathrm{s}}=2\right)$ were determined in the same way as in the paper [12]. The parameter $q(N, d)$ was calculated for a given thickness $d$ of adsorbate layer and for a given number of adatoms $N$ per unit area of substrate from the minimization condition (Eqs. $(12,13)$ )

$$
\left.\left.\frac{\partial \sigma\left(f_{2}(q)\right)}{\partial q}\right|_{N, d} \equiv \frac{\partial Q(q)}{\partial q}\right|_{N, d} .
$$

*Atomic units (a. u.) are used unless otherwise stated. 


\section{Results and discussion}

The SEDP (Eqs. $(2-5,9))$ is determined by the three independent parameters: $\gamma\left(r_{\mathrm{s}}\right), b\left(r_{\mathrm{s}}\right), q(N, d)$. Parameters $\gamma\left(r_{\mathrm{s}}\right)$ and $b\left(r_{\mathrm{s}}\right)$ characterize the substrate and for $r_{\mathrm{s}}=2$ their values are equal to 0.9742801 and 0.44017974 respectively. For a given adsorbate (defined by the value of $d$ and by the surface concentration $N^{\mathrm{m}}$ of the alkali metal monolayers, calculated from Eqs. (11, A1.2)), the values of the parameter $q$ as a function of $N$ are collecied in the Table.

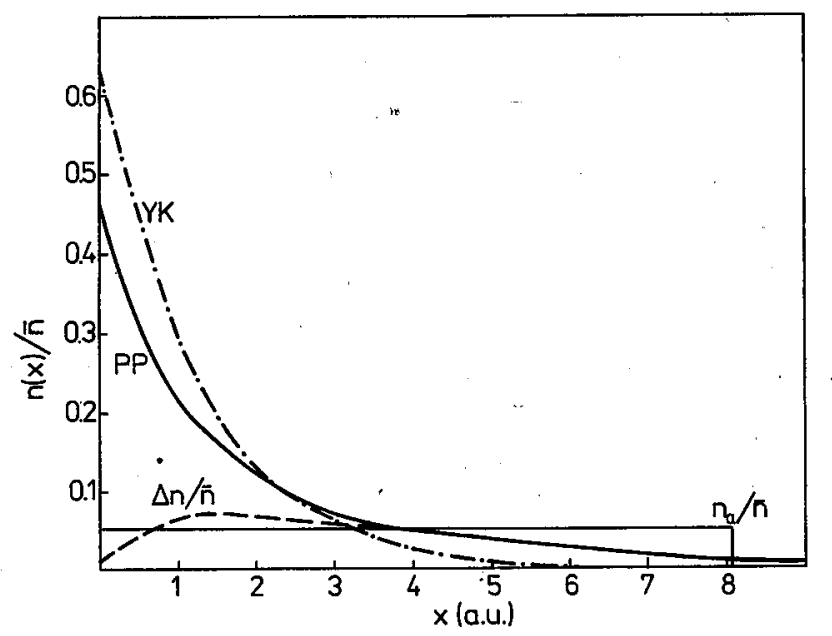

Fig. 3. The SEDP versus the distance $x$ for a cesium monolayer on the metal with $r_{\mathrm{s}}=2$. The solid curve presents the SEDP, $n\left(x, N^{\mathrm{m}}\right)$, the dashed curve - the electron density profile $\Delta n\left(x, N=N^{\mathrm{m}}\right)$ of an isolated monolayer and the dashed-dotted curve the SEDP $n(x)$ calculated by the use of YK model [3].

For verification of the presented model we employ the WF dependence on a kind of adlayer. To be consistent with the Lang calculations [6] we have used the expression of Koopmans [13] for WF, namely

$$
\varphi=\Delta \Phi-\frac{1}{2} k_{\mathrm{F}}^{2}-\mu_{\mathrm{xc}}
$$

where

$$
\Delta \Phi=4 \pi \int_{-\infty}^{+\infty} x n_{\mathrm{t}}(x) \mathrm{d} x
$$

is the surface dipole barrier and $\mu_{\mathrm{xc}}$ is the exchange and

corelation part of the chemical potential of a uniform electron gas $[1,10]$.

The change of WF, $\Delta \varphi(N)$, due to adsorption is equal to

$$
\Delta \varphi(N, d)=\varphi(N, d)-\varphi(0,0)=\Delta \Phi(N, d)-\Delta \Phi(0,0),
$$


TABLE

The values of the parameter $q(N, d)$ for alkali metal submonolayers $d$ are in atomic units.

\begin{tabular}{|c|c|c|c|c|c|}
\hline \multirow{3}{*}{$N$} & \multicolumn{5}{|c|}{$q(N, d)$} \\
\hline & $d_{\mathrm{Li}}=4.68$ & $d_{\mathrm{Na}}=5.72$ & $d_{\mathrm{K}}=7.13$ & $d_{\mathrm{Rb}}=7.57$ & $d_{\mathrm{Cs}}=8: 08$ \\
\hline & $N^{\mathrm{m}}=11.56$ & $N^{\mathrm{m}}=8.03$ & $N^{\mathrm{m}}=5.29$ & $N^{\mathrm{m}}=4.56$ & $N^{\mathrm{m}}=3.87$ \\
\hline .25 & 3.0181079 & 3.0063574 & 2.9956741 & 2.9934553 & 2.9905028 \\
\hline .50 & 2.2580932 & 2.2218141 & 2.1866514 & 2.1790528 & 2.1687720 \\
\hline .75 & 1.8305172 & 1.7550083 & 1.6724086 & 1.6529449 & 1.6254828 \\
\hline 1.00 & 1.5447972 & 1.4265152 & 1.2813421 & ].2438112 & 1.1879887 \\
\hline 1.25 & 1.3497855 & 1.2021055 & 1.0166099 & .9682663 & .8964695 \\
\hline 1.50 & 1.2153660 & 1.0540217 & .8578023 & .8090419 & .7396370 \\
\hline 1.75 & 1.1203916 & .9548905 & .7632229 & .7181792 & .6561718 \\
\hline 2.00 & 1.0511536 & .8863258 & .7039922 & .6627982 & .6070334 \\
\hline 2.25 & .9991719 & .8372302 & .6644259 & .6263212 & .5751466 \\
\hline 2.50 & .9591343 & .8008893 & .6364472 & .6007141 & .5529071 \\
\hline 2.75 & .9276037 & .7731708 & .6157401 & .5818353 & .5365592 \\
\hline 3.00 & .9022871 & .7514700 & .5998543 & .5673847 & .5240599 \\
\hline 3.25 & .8816103 & .7340969 & .5873129 & .5559897 & .5142079 \\
\hline 3.50 & .8644696 & .7199201 & .5771795 & .5467893 & .5062530 \\
\hline 3.75 & .8500719 & .7081614 & .5688341 & .5392150 & .4997029 \\
\hline 4.00 & .8378385 & .6982710 & .5618515 & .5328783 & .4942207 \\
\hline 4.25 & .8273362 & .6898499 & .5559293 & .5275041 & \\
\hline 4.50 & .8182375 & .6826036 & .5508479 & .5228934 & \\
\hline 4.75 & .8102916 & .6763101 & .5464445 & .5183968 & \\
\hline 5.00 & .8033010 & .6707988 & .5425949 & & \\
\hline 5.25 & .7971105 & .6659377 & .5392031 & & \\
\hline 5.50 & .7915969 & .6616213 & .5361936 & & \\
\hline 5.75 & .7866591 & .6577662 & & & \\
\hline 6.00 & .7822156 & .6543045 & & & \\
\hline 6.25 & .7781990 & .6511823 & & & \\
\hline 6.50 & .7745535 & .6483520 & & & \\
\hline 6.75 & .7712325 & .6457774 & $r$ & & \\
\hline 7.00 & .7681969 & .6434268 & & & \\
\hline 7.25 & .7654119 & .6412725 & & & \\
\hline 7.50 & .7628511 & .6392925 & & & \\
\hline 7.75 & .7604893 & .6374669 & & & \\
\hline 8.00 & .7583040 & .6357798 & & & \\
\hline 8.25 & .7562804 & .6342162 & & & \\
\hline 8.50 & .7543993 & & & & \\
\hline 8.75 & .7526480 & & & & \\
\hline 9.00 & .7510151 & & & & \\
\hline 9.25 & .7494887 & & & & \\
\hline 9.50 & .7480597 & & & & \\
\hline 9.75 & .7467198 & & & & \\
\hline 10.00 & .7454613 & & & & \\
\hline
\end{tabular}


where $\varphi(0,0), \Delta \Phi(0,0)$ are WF and the surface dipole barrier of the clean substrate respectively.

Using the model of SEDP, given in Section 2, we obtain

$$
\Delta \varphi(N, d)=\frac{81.64}{r_{\mathbf{s}}^{3}} s(N) d\left(\frac{2}{q(N, d)}+x_{0}-\frac{d}{2}\right) \mathrm{eV} .
$$

For a given adsorbate (fixed $d) \Delta \varphi$ as a function of adsorbate concentration $N$ has a minimum at $N=N_{\mathrm{m}}$

$$
\frac{\partial(\Delta \varphi(N, d)}{\partial N} \|_{N=N_{\mathbf{m}}(d)}=0 .
$$

The dependences $\Delta \varphi_{\mathrm{m}}(d)=\Delta \varphi\left(N=N_{\mathrm{m}}, d\right)$ and $N_{\mathrm{m}}(d)$, obtained from presented model and from models given by other authors, are shown in Figs. 1 and 2, respectively. Figure 1 contains additionaly the experimental data.

It is seen in Fig. 1 that our model gives reasonable good agreement with the Lang's results and also with the experimental data. From Fig. 2 it follows that this model gives the same trend of the function $N_{\mathrm{m}}(d)$ as calculated by Lang[6]. The same trend is also observed experimentally $[14,15]$ in contradiction to the results obtained by Yamauchi and Kawabe [3], and by Bigun [4].

The numerical value of $\Delta \varphi_{\mathrm{m}}(N)$, as well as the value of $N_{\mathrm{m}}$ calculated by Yamauchi and Kawabe (YK) [3] for a sodium adlayer on the metal substrate with $r_{\mathrm{s}}=2$, are different from these calculated in the present paper. Namely, Yamauchi and Kawabe gave values of $\Delta \varphi_{\mathrm{m}}(N)$ and $N_{\mathrm{m}}:-1.9 \mathrm{eV}$ and 2.4, respectively. For the same system we have found that $\Delta \varphi_{\mathrm{m}}(N)=-2.25 \mathrm{eV}, N_{\mathrm{m}}=3.73$. This discrepancy follows, probably, from the fact that the surface energy has a very shallow minimum and only the deeper numerical analysis of the problem has brought the results quoted above.

Figure 3 presents the SEDP for cesium monolayer on $r_{\mathrm{s}}=2$ substrate. The $\Delta n(x) / \bar{n}$ curve is also given representing the EDP of the isolated adlayer, where $\Delta n\left(x, N=N^{\mathrm{m}}\right)=n\left(x, N^{\mathrm{m}}\right)-n(x, 0)$.

The analysis of $\Delta n(x, N)$ as a function of $x$ shows that maximum value of this function increases with $N$ and its position moves to the center of the adlayer. Therefore in comparison with the YK and B models, the SEDP calculated from our model better balances the positive charge distribution in the adlayer.

In spite of relative simplicity of the presented model, it gives results comparable with Lang's self-consistent calculations and can be used for an analytical determination of many surface properties of metals such as, for example, work function, surface energy, heat of adsorption, and so on.

\section{Appendix 1}

For the description of the coverage of an adsorbate, two scales are used. Namely: $N$ - the number of adatoms per unit area of an adsorbent (Eq. (10)) and the degree of surface coverage $\theta$. The $\theta$ scale was employed earlier (see e.g.[14, 
16]) and recently rather the $N$ scale is used [3-6]. The both scales, within the jellium model, are connected by the relation:

$$
N=\frac{s d}{0.01172 r_{\mathrm{s}}^{3}}=\frac{\theta d}{0.01172 r_{\mathrm{sa}}^{3}}, \quad 0 \leq \theta \leq 1,
$$

where $r_{\mathrm{s}}$ and $r_{\mathrm{sa}}$ are the Wigner-Seitz radii of the bulk substrate and of the bulk adsorbate, respectively.

The above relation follows from the definitions of the quantities $N_{\mathrm{a}}$ and $s$ (see Eqs. $(10,11)$ ), and the quantity $\theta$ is defined as

$$
\theta=\frac{n_{\mathrm{a}}}{n_{\mathrm{a}}^{\mathrm{m}}}=\frac{N}{N^{\mathrm{m}}}
$$

where $n_{\mathrm{a}}^{\mathrm{m}}$ is the electron density in the monolayer and $N^{\mathrm{m}}-$ the number of adatoms per unit area in the monolayer.

From Eq. (A1.1) it is seen that for $\theta=1$ we obtain different values of $N^{m}$ for different kind of adatoms (see Table), therefore $N$ is better scale than $\theta$.

\section{Appendix 2}

$$
\begin{gathered}
\Gamma(\gamma)=-\frac{3}{c \alpha}\left(\frac{A}{r_{\mathrm{s}}^{2}} c_{1}-\frac{B}{r_{\mathrm{s}}} c_{2}-\frac{C}{D} h\right) \\
+\frac{1.5}{r_{\mathrm{s}}^{3}} \times \frac{1}{3}(1-s) s^{2} d^{3}+\left(s d-x_{0}\right)\left[\frac{1}{3}\left(s d-x_{0}\right)^{2}-\frac{2 b_{1}}{c^{3}}\right] \\
+\frac{b_{1}^{2}}{2 c^{3}}+\frac{b^{2}}{2 c^{3} \alpha^{3}}+\frac{2 b_{1}}{c^{3}}\left\{1-(1-s) \exp \left(c x_{0}\right)-s \exp \left[-c\left(d-x_{0}\right)\right]\right\} \\
-\frac{1}{72} c \alpha\left(b+\ln b_{1}\right)
\end{gathered}
$$

and

$$
\begin{gathered}
Q(q)=\frac{3}{r_{\mathrm{s}}^{3}} s d\left\{\frac{2 x_{0}-1.375 s d}{q}\right. \\
\left.+\frac{3-(1-s)\left(3-q x_{0} e^{q x_{0}}\right)-s\left[3+q\left(d-x_{0}\right) e^{-q\left(d-x_{0}\right)}\right]}{q^{2}}+\frac{b_{1}(c+2 q)}{c(c+q)^{2}}\right\} \\
+\int_{0}^{\infty}\left\{y^{3}(y-1)\left[\frac{A}{r_{\mathrm{s}}^{2}} y+\frac{A}{r_{\mathrm{s}}^{2}}-\frac{B}{r_{\mathrm{s}}}-\frac{C r_{s}}{D\left(D+r_{s}\right)(y+\varrho)}\right]+\frac{1}{72} \frac{z^{2}}{y^{3}}\right\} \mathrm{d} x \\
y=\left(b_{1} e^{-c x}+s x d q^{2} e^{-q x}\right)^{1 / 3} \\
z=c b_{1} e^{-c x}+(q x-1) s d q^{2} e^{-q x} .
\end{gathered}
$$

The other symbols appearing in Eqs. (A2.1-A2.4), have the following meaning: the constants $A, B, C$, and $D$ are equal to $1.105,0.4581,0.44$ and 7.8 , respectively. The parameters $c, \alpha$, and $b_{1}$ are defined by Eqs. (4b), (4c), and (4a).

The quantites $c_{1}, c_{2}$ and $h$ are given by the following expressions: 


$$
\begin{gathered}
c_{1}=\frac{11+10 b_{1}}{3}-\frac{5+2 b_{1}}{10} y^{2}-u+v \\
c_{2}=\frac{11+4 b_{1}}{12}-\frac{4+b_{1}}{4} y_{0}-u-v \\
h=\varrho\left\{\frac{b}{3(1+\varrho)}-\left(1-y_{0}\right)\left(\frac{1+y_{0}}{2}-\varrho\right)\right. \\
\left.+\frac{1}{1+\varrho^{3}}\left[u-v-\varrho(u+v)-\varrho^{5} \ln \frac{1+\varrho}{y_{0}+\varrho}\right]\right\}
\end{gathered}
$$

where

$$
\begin{gathered}
u=\frac{1}{2} \ln \frac{3\left(1-y_{0}\right)}{b}, \\
v=\frac{1}{\sqrt{3}} \operatorname{arctg}\left(\frac{1}{\sqrt{3}} \frac{1-y_{0}}{1+y_{0}}\right)
\end{gathered}
$$

and

$$
\begin{gathered}
y_{0}=b_{1}^{1 / 3}, \\
\varrho=\frac{r_{\mathrm{s}}}{D} .
\end{gathered}
$$

\section{References}

[1] N.D. Lang, W. Kohn, Phys. Rev. B 1, 4555 (1970).

[2] J.P. Perdew, Phys. Rev. $B$ 21, 869 (1980) (see also references therein).

[3] H. Yamauchi, U. Kawabe, Phys. Rev. B 14, 2687 (1976).

[4] G.J. Bigun, Ukr. Fiz. Zh. 24, 1313 (1979).

[5] G.J. Bigun, I.D. Nabitovitch, Fiz. Tverd. Tela 21, 1349 (1979).

[6] N.D. Lang, Phys. Rev. B 4, 4234 (1971).

[7] K. F. Wojciechowski, Surf. Sci. 55, 246 (1976).

[8] R. Smoluchowski, Phys. Rev. 60, 661 (1941).

[9] J.P. Perdew, Phys. Rev. B ,21, 869 (1980).

[10] N.D. Lang, Solid Slate Phys. 28, 225 (1975).

[11] A. Kiejna, Phys. Slatus Solidi B 105, 147 (1981).

[12] J.M. Rogowska, K.F. Wojciechowski, II. Bogdanów, Acla Phys. Pol. A78, 651 (1990).

[13] N.D. Lang, W. Kohn, Phys. Rev. B 3, 1215 (1971).

[14] Z. Sidorski, J. Pelly, R. Gomer, J. Chem. Phys. 20, 2383 (1969).

[15] R. Błaszczyszyn, M. Błaszczyszyn, R. Męclewski, Surf. Sci. 51, 396 (1975).

[16] A.G. Fedorus, A.G. Naumovets, Surf. Sci. 21, 426 (1970). 